\title{
A Comparative Study of Serum Cystatin C with Serum Creatinine as an Early Marker of Acute Renal Dysfunction in Intensive Care Patients
}

\author{
Arun Sinha ${ }^{1}$, Vibha $\mathrm{C}^{2}, \mathrm{HL}$ Vishwanath ${ }^{3}$, B Prabhakar $^{4}$
}

\begin{abstract}
Introduction: Acute kidney injury (AKI) is a very common complication occurring in medical intensive care, causing significant morbidity and mortality. Several biomarkers have been studied for their utility in diagnosing acute renal failure but without much success. Still, serum creatinine is the marker of choice despite having several shortcomings. In this study, we have tried to see the usefulness of serum cystatin $C$ in comparison to serum creatinine as an early marker of AK.

Materials and methods: A cross-sectional study was conducted involving 50 cases admitted in the ICU of our hospitals and their Cystatin C and creatinine was estimated. The results were compared with cystatin c and creatinine values of healthy controls.

Results: In cases, the mean cystatin C was found to be $744.58 \pm 321.00 \mathrm{ng} / \mathrm{mL}$. Data showed that $50 \%$ of cases had abnormal values for serum cystatin C. Estimation of serum creatinine in cases showed a value of $1.07 \pm 0.45 \mathrm{mg} / \mathrm{dL}$ with a $p=0.008$. Among cases, $20 \%$ of patients had abnormal creatinine. Receiver operator curve (ROC) analysis with AUC showed that cystatin $C$ with a cutoff $>40$ had sensitivity and specificity of $100 \%$ and AUC $1.000(p=1.0)$ the sensitivity and specificity were 50 and 70\%, respectively, and AUC $0.637(p=0.013)$. The creatinine clearance as estimated by both MDRD and CKD EPI formulae were found to be normal in both cases and controls.

Conclusion: The results clearly showed that estimation of serum cystatin c in ICU patient could go long way in reducing mortality due to acute renal dysfunction.

Keywords: MDRD, Renal function, Renal function test, ROC curve.

Indian Journal of Medical Biochemistry (2020): 10.5005/jp-journals-10054-0140
\end{abstract}

\section{INTRODUCTION}

Acute kidney injury (AKI) is characterized by a rapid decline in glomerular filtration rate (GFR) over hours to days. ${ }^{1}$ Acute renal failure complicates up to $30 \%$ of the intensive care unit (ICU) admissions with a very high mortality rate. ${ }^{1,2}$ Acute kidney injury is generally asymptomatic and is diagnosed when biochemically there is an elevation of blood urea nitrogen (BUN) and serum creatinine. Retention of nitrogenous waste products, oliguria (urine output $<400 \mathrm{~mL} /$ day), electrolyte, and acid-base abnormalities are frequent clinical manifestations. Acute kidney injury is often considered reversible. It is further classified as (a) prerenal (55\%), (b) renal or intrinsic ( $40 \%)$, and (c) postrenal (5\%) depending upon the etiology. ${ }^{1}$ The first step in evaluating a patient with renal failure is to ascertain whether it is acute or chronic. Acute kidney injury is associated with a rapid increase in serum creatinine and $B U N$ values within 24-48 hours that parallel to the change in the hemodynamic status of the patients. Several criteria have been introduced to assess the severity of AKI. These include the risk, injury, failure, loss, ESRD (RIFLE) criteria, acute kidney injury network (AKIN), and kidney disease improving global outcomes (KADIGO). The populations of patients admitted in the ICU belong to both surgical and nonsurgical subset. In the surgical population, a very high proportion is contributed by patients who have undergone cardiac interventions, though a lower incidence of AKI is also seen in elective surgical patients. There is a higher incidence of AKI in sepsis patients. The incidence of contrast-induced AKI is less (11.5-19\% of all admissions) than seen in the ICU population at large. ${ }^{3}$ Early diagnosis of AKI in these ICU patients can substantially reduce mortality and in this \begin{tabular}{l}
\hline${ }^{1-3}$ Department of Biochemistry, Bangalore Medical College and \\
Research Institute, Bengaluru, Karnataka, India \\
${ }^{4}$ Department of Internal Medicine, Bangalore Medical College and \\
Research Institute, Bengaluru, Karnataka, India \\
Corresponding Author: Arun Sinha, Department of Biochemistry, \\
Bangalore Medical College and Research Institute, Bengaluru, \\
Karnataka, India, Phone: +919916106255 , e-mail: sinhadrarun7@ \\
gmail.com \\
How to cite this article: Sinha A, Vibha C, Vishwanath HL, et al. A \\
Comparative Study of Serum Cystatin C with Serum Creatinine as an \\
Early Marker of Acute Renal Dysfunction in Intensive Care Patients. \\
Indian J Med Biochem 2020;24(3):115-118. \\
Source of support: Nil \\
Conflict of interest: None \\
\hline \hline
\end{tabular}

endeavor serum cystatin C has come up as a sensitive and novel biomarker. The main objective of this study is to highlight the fact that the uses of novel biomarkers like cystatin C go a long way in identifying patients at risk of developing AKI and subsequently reduce mortality in the ICU (Tables 1 to 3 ).

\section{Materials and Methods}

The study done was a cross-sectional case-control study on patients admitted in the ICU of Bowring and Lady Curzon Hospital along with Victoria Hospital of Bengaluru Medical College and Research Institute. The sample size was 50 cases and 50 age- and sex-matched controls with the power of the study being 1.000. The institutional 
ethical clearance board approved the study. After taking informed consent from the patients/attendants, $5 \mathrm{~mL}$ of venous blood was drawn by venipuncture. Also, a spot urine sample was taken for the calculation of creatinine clearance. Blood was centrifuged and serum was separated. The separated serum was stored at $-20^{\circ} \mathrm{C}$ till the tests would be performed. Creatinine clearance was calculated using the modification of diet in renal disease (MDRD) and chronic kidney disease epidemiology collaboration (CKD EPI) formulae. The serum samples of the patients were obtained preferably within the first 24 hours of admission to the ICU. None of the patients selected had any history of previous renal disease. Patients between the age group 35 years and 60 years were chosen. Pregnant and lactating females and those patients who had documented evidence of having taken any nephrotoxic drugs in the last week were excluded from the study. The serum samples were run on Beckman Coulter AU 560 autoanalyzer for urea and creatinine. The estimation of cystatin C was done using a 96-well coated ELISA kit obtained from Biovendor. The results were expressed on continuous measurements and were presented as mean \pm SD (Min-Max) and results on categorical measurements were presented in number (\%). Significance was assessed at a $5 \%$ level of significance. The following assumptions on data were made: (1) Dependent variables should be normally distributed; (2) Samples drawn from the population should be random.

Student's t-test (two-tailed, independent) was used to find the significance of study parameters on a continuous scale between two groups (intergroup analysis) on metric parameters. Leven 1s test for homogeneity of variance was performed to assess the

Table 1: Age distribution of patients studied

\begin{tabular}{lrrrrr}
\hline & \multicolumn{3}{c}{ Control } & & \multicolumn{2}{c}{ Case } \\
\cline { 2 - 3 } \cline { 5 - 6 } Age in year & No & $(\%)$ & & No & $(\%)$ \\
\hline $20-30$ & 5 & 10 & & 10 & 20 \\
$31-40$ & 19 & 38 & & 13 & 26 \\
$41-50$ & 7 & 14 & & 7 & 14 \\
$51-60$ & 10 & 20 & & 9 & 18 \\
$61-70$ & 7 & 14 & & 11 & 22 \\
Total & 50 & 100 & & 50 & 100 \\
Mean \pm SD & \multicolumn{3}{c}{$46.80 \pm 13.13$} & & $47.06 \pm 14.08$
\end{tabular}

Samples are age-matched with $p=0.783$

Table 2: Gender distribution of patients studied

\begin{tabular}{llcccc}
\hline & \multicolumn{3}{c}{ Control } & & \multicolumn{2}{c}{ Case } \\
\cline { 2 - 3 } \cline { 5 - 6 } Gender & No & $(\%)$ & & No & $(\%)$ \\
\hline Female & 17 & 34 & & 16 & 32 \\
Male & 33 & 66 & & 34 & 68 \\
Total & 50 & 100 & & 50 & 100 \\
\hline
\end{tabular}

Samples are gender matched with $p=0.832$

Table 3: Serum creatinine $(\mathrm{mg} / \mathrm{dL})$ distribution of patients studied

\begin{tabular}{lrrrrr}
\hline $\begin{array}{l}\text { Serum } \\
\text { creatinine }\end{array}$ & \multicolumn{3}{c}{ Control } & & \multicolumn{2}{c}{ Case } \\
\cline { 2 - 3 } \cline { 5 - 6 }$(\mathrm{mg} / \mathrm{dL})$ & No & $(\%)$ & & No & (\%) \\
\hline Normal & 44 & 88 & & 40 & 80 \\
Abnormal & 6 & 12 & & 10 & 20 \\
Total & 50 & 100 & & 50 & 100 \\
\hline
\end{tabular}

$p=0.275$, not significant, Chi-square test homogeneity of variance. Chi-square/Fisher's exact test was used to find the significance of study parameters on a categorical scale between two or more groups. Receiver operator curve (ROC) curve analysis was employed to define the markers. The Statistical software namely SAS 9.2, SPSS 15.0, Stata 10.1, MedCalc 9.0.1, Systat 12.0, and R environment ver.2.11.1 was used for the analysis of the data (Tables 4 to 6).

Cystatin C in cases was estimated to be $744.58 \pm 321.00 \mathrm{ng} /$ $\mathrm{mL}$ and in controls $21.26 \pm 7.63 \mathrm{ng} / \mathrm{mL}$ with $p<0.001$. Estimation of serum creatinine in cases showed a value of $1.07 \pm 0.45 \mathrm{mg} / \mathrm{dL}$ and in controls, $0.85 \pm 0.34 \mathrm{mg} / \mathrm{dL}, p=0.008$. ROC analysis with area under curve (AUC) showed that cystatin $C$ with a cutoff $>40$ had a sensitivity and specificity of $100 \%$ and AUC $1.000(p<0.001)$. For creatinine with cutoff $>1.0$, the sensitivity and specificity were 50 and $70 \%$, respectively, and AUC 0.637 ( $p=0.013)$. The creatinine clearance as estimated by both MDRD and CKD EPI formulae were found to be normal in both cases and controls.

\section{Results}

The current cross-sectional study in which the serum creatinine and serum cystatin $C$ values were measured and compared between 50 patients admitted in the ICU with the age- and sex-matched normal subjects taken as controls. The result was tabulated and statistically analyzed. In this study, the age of the cases ranged from 30 to 65 years which was similar to the control $(46.80 \pm 13.13$ and $47.06 \pm$ $14.08, p=0.732$ ). Gender wise among cases $34 \%$ of subjects were females and $66 \%$ were males, $p=0.832$. In comparison among the controls, $32 \%$ were females and $68 \%$ were males, $p=0.832$ which is statistically non-significant. This finding was in accordance with the study conducted by Herget-Rosenthal et al. ${ }^{4}$ where there was no correlation drawn with the gender of the patients.

The analysis of creatinine among cases showed that only $20 \%$ had abnormal values in comparison to $80 \%$ who had normal values. Among subjects taken as control, $12 \%$ had abnormal values with, $p=0.275$. This shows that there was no difference in creatinine measurement in cases and controls. Urea estimation indicated that

Table 4: Cystatin C (ng/mL) distribution of patients studied

\begin{tabular}{lrcrrr}
\hline \multirow{2}{*}{$\begin{array}{l}\text { Cystatin } C \\
(\mathrm{ng} / \mathrm{mL})\end{array}$} & \multicolumn{3}{c}{ Control } & & \multicolumn{2}{c}{ Case } \\
\cline { 2 - 3 } \cline { 5 - 6 } \cline { 5 - 6 } & No & $\%$ & & No & $\%$ \\
\hline Normal & 45 & 90 & & 0 & 32 \\
Abnormal & 5 & 10 & & 100 & 68 \\
Total & 50 & 100 & & 100 & 100 \\
\hline
\end{tabular}

$p<0.001$, statistical significance of cystatin c measured in cases and controls, Chi-square test

Table 5: Comparison of study outcome variables in the two groups studied

\begin{tabular}{lccl}
\hline & Control & Case & pvalue \\
\hline $\begin{array}{l}\text { Serum creatinine } \\
\text { (mg/dL) }\end{array}$ & $0.85 \pm 0.34$ & $1.07 \pm 0.45$ & $0.008^{*}$ \\
$\begin{array}{l}\text { Cystatin C (mg/dL) } \\
\text { Creatinine clearance } \\
\text { by MDRD (mL/ }\end{array}$ & $166.33 \pm 6.68$ & $744.58 \pm 321.00$ & $<0.001^{*}$ \\
$\begin{array}{l}\text { minute) } \\
\begin{array}{l}\text { Creatinine clearance } \\
\text { by CKD-EPI }\end{array}\end{array}$ & $99.52 \pm 30.68$ & $79.22 \pm 39.96$ & $0.008^{*}$ \\
\hline
\end{tabular}

${ }^{*} p<0.05$ 
Table 6: ROC curve analysis to assess the early markers for acute renal dysfunction

\begin{tabular}{|c|c|c|c|c|c|c|c|c|}
\hline & Cutoff & Sensitivity & Specificity & $L R+$ & $L R-$ & $A \cup C$ & $S E$ & $p$ value \\
\hline $\begin{array}{l}\text { Serum creatinine } \\
(\mathrm{mg} / \mathrm{dL})\end{array}$ & $>1.0$ & 50 & 70 & 2.5 & 0.62 & 0.637 & 0.055 & $0.013^{*}$ \\
\hline Cystatin C (mg/dL) & $>40$ & 100 & 100 & 50 & 0.002 & 1 & 0 & $<0.001$ \\
\hline $\begin{array}{l}\text { Creatinine clearance by } \\
\text { MDRD ( } \mathrm{mL} / \text { minute) }\end{array}$ & $<73$ & 54.17 & 76.00 & 2.26 & 0.6 & 0.649 & 0.055 & $0.007^{* *}$ \\
\hline $\begin{array}{l}\text { Creatinine clearance by } \\
\text { CKD-EPI }\end{array}$ & $<89$ & 62.5 & 66.00 & 1.84 & 0.57 & 0.634 & 0.056 & $0.017^{*}$ \\
\hline
\end{tabular}

$*{ }^{* * *} p<0.05$

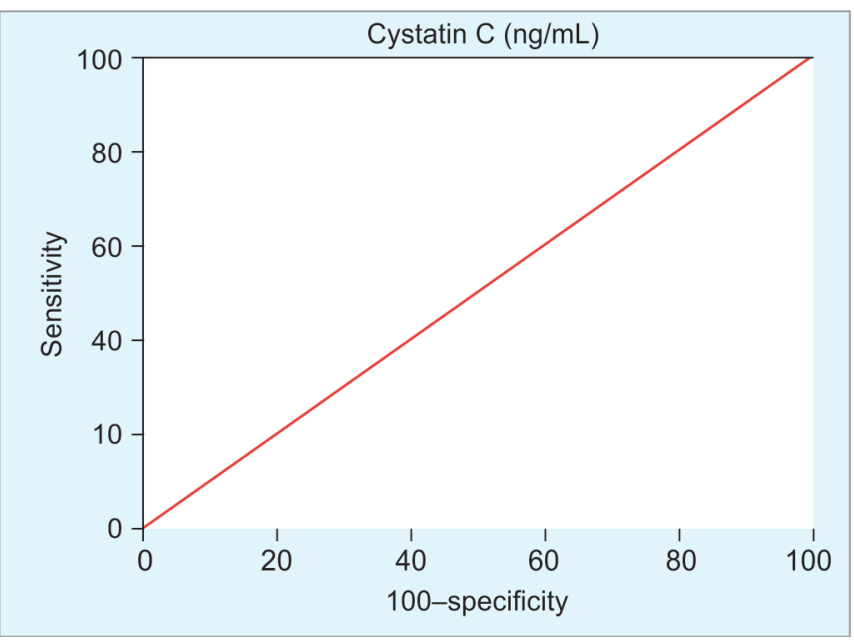

Fig. 1: ROC curve of creatinine

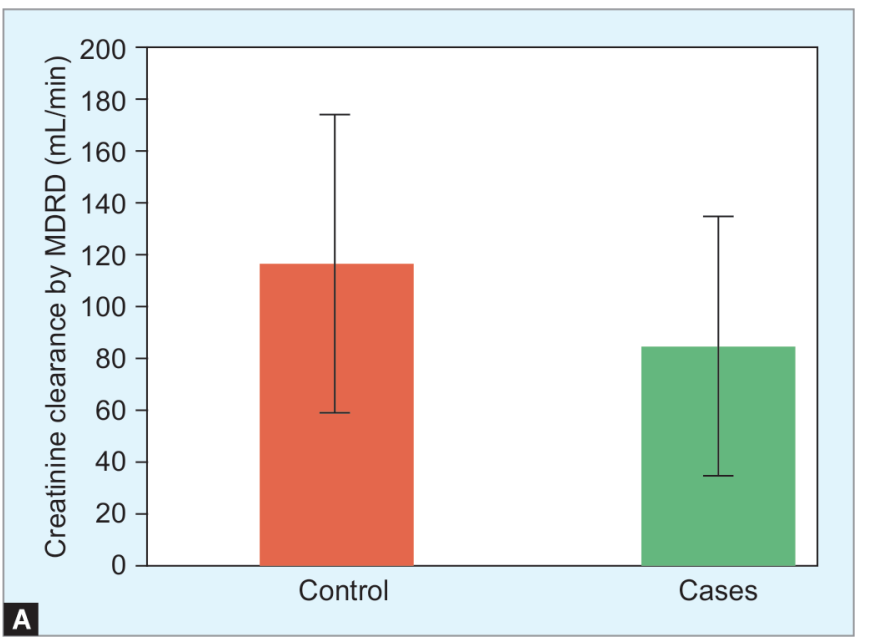

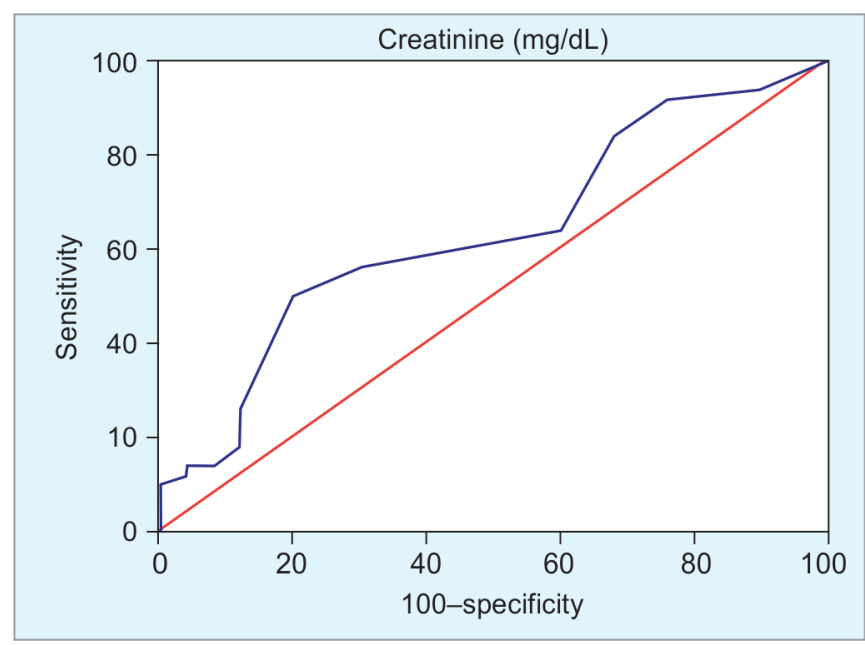

Fig. 2: ROC curve of cystatin C

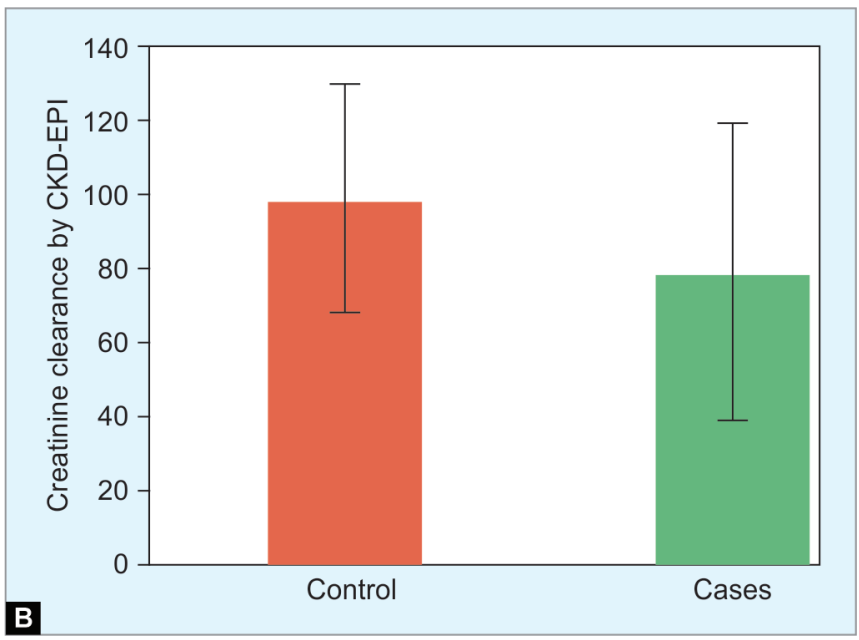

Figs $3 \mathrm{~A}$ and $\mathrm{B}$ : Creatinine clearance calculated by MDRD and CKD-EPI in both cases and controls

both the groups, i.e., cases and controls had values in the normal range. (Mean \pm SD for cases, $34.38 \pm 16.76$, controls $33.48 \pm 13.23$, $p=0.766$, not significant) indicating that there was no significant difference in urea values. The literature also says that urea levels can be affected by several non-renal factors and that a high value does not confirm renal failure nor a normal value rule it out. ${ }^{5}$

Cystatin C evaluation showed that $100 \%$ of cases had abnormal values in contrast to controls where only $10 \%$ of subjects had abnormal values with $p<0.001$. ROC analysis showed that the sensitivity and specificity for serum cystatin C was 100\% $(p<0.001)$ as compared to creatinine which had a sensitivity and specificity of 50 and $70 \%$, respectively, with $p=0.013$ (Figs 1 to 3 ).

\section{Discussion}

Creatinine is the most widely used biomarker for the assessment of kidney function. It is (MW $113 \mathrm{Da}$ ) cyclic anhydride of creatine that is the final product of decomposition of phosphocreatine. The amount of creatinine produced each day is constant and is related to muscle mass. Diet also influences the level of 
creatinine. ${ }^{5}$ The important thing to note is that even though there is no significant reabsorption of creatinine by the renal tubules, there is a small but significant tubular section. This gets increased in cases where there is renal dysfunction resulting in underestimating the values, thus providing a wrong picture. ${ }^{5}$ Because of its constant rate of production and maintenance of its plasma concentration within a narrow range by glomerular filtration, it is widely used as a marker for acute and chronic renal dysfunction. But a major drawback is that a normal serum creatinine does not rule out the presence of renal impairment. Cystatin C is a 120 -amino acid, $12.8 \mathrm{kDa}$ protein that is a member of the family of competitive inhibitors of lysosomal cysteine proteinases. It is the most abundant extracellular cysteine protease inhibitor. It is synthesized by all nucleated cells. It is a non-glycosylated protein. It is freely filtered through the normal glomerular membrane and almost completely reabsorbed and catabolized by the proximal tubules. With regards to its renal function, its most important attributes are small size and high isoelectric point ( $\mathrm{PI}=9.2$ ) which enables it to be freely filtered. The cystatin $C$ concentration is independent of muscular mass, inflammatory diseases, sex, age, or diet..$^{6-12}$ It is also sensitive to the changes in the creatinine blind range of GFR $(40-70 \mathrm{~mL} /$ minute $/ 1.73 \mathrm{~m}^{2}$ ). Since there is no tubular secretion of cystatin $C$, it is a very sensitive indicator of minor changes in the GFR in the earliest stages of kidney disorders. ${ }^{13-17}$

\section{Conclusion}

In our study, it was found that there was a significant elevation in serum cystatin $C$ levels in cases compared to controls. Serum creatinine was found to be normal in both cases and controls. This study suggests that despite the serum creatinine is normal, the serum cystatin C was elevated with $100 \%$ sensitivity and specificity in cases. Also, all the cases had normal creatinine clearance, signifying the fact that cystatin C can be used as a marker for early diagnosis of acute renal failure and identifying patients at risk in the ICU. Our study has results that are comparable to other studies done worldwide.

\section{SUMmARY}

Despite all the drawbacks, creatinine is still the marker of choice because of the lower costs involved in the estimation. Even though our study shows a clear advantage of cystatin $C$ in comparison to creatinine estimation but still more work needs to be done to come up with methods that can be utilized to estimate cystatin $C$ which is economically comparable to creatinine estimation. This will enable us to replace creatinine with more sensitive parameters which in turn help us reduce mortality in AKI cases.

\section{References}

1. Liu DK, Chertow GM. Acute renal failure Fauci AS, Kasper DL, Longo DL, et al., ed. Harrison's Principles of Internal Medicine. 17th ed., New York: McGraw Hill, Health Professional Division; 2008. 1752-1761.

2. Brady HR. Acute renal failure. Brenner and Rector's The Kidney. 7th ed., Philadelphia: Saunders; 2004.

3. Lameiere N. The path physiology of acute renal failure. Crit Care Clin 2005;21(2):197. DOI: 10.1016/j.ccc.2005.01.001.

4. Herget-Rosenthal S, Marggraf G, Hüsing J, et al. Early detection of acute renal failure by serum cystatin C. Kidney Int 2004;66(3): 1115-1122. DOI: 10.1111/j.1523-1755.2004.00861.x.

5. Burtis CA, Ashwood ER, Bruns DE. Tietz Textbook of Clinical Chemistry and Molecular Diagnostics. 4th ed., St. Louis, Missouri: Elsevier Saunders; 2006. 546, 602, 607, 612, 755, 774, 797, 801, 837 \& 1193. 15 Proc Nadl Acad Sci 1986 Nov; 83:8749-53.

6. Vasudevan DM, Sreekumari S, Vaidyanathan K, Cystatin C as a filtration marker, Textbook of Biochemistry for Medical Students. 6th ed., Kochi, St. Louis, Panama City, London, New Delhi, Ahmadabad, Bengaluru, Chennai, Hyderabad.

7. Kolkata, Lucknow, Mumbai, Nagpur, Jaypee Brothers Medical Publishers (P) Ltd.2011; p. 322.

8. Bhagavan NV, Ha C-E. Creatine and related compounds; Essentials of Medical Biochemistry with Clinical Cases. 1st ed, Amsterdam, Boston, Heidelberg, London, Oxford, Paris, San Diego, San Francisco, Singapore, Sydney Tokyo: Elseveir Academic Press; 2011. 181-182.

9. Ekiel I, Abrahamson M, Fulton DB, et al. NMR structural studies of human cystatin C dimmers and monomer. J Mol Biol 1997;271(2): 266-277. DOI: 10.1006/jmbi.1997.1150.

10. Marin T, DeRossett B, Bhatia J. Urinary biomarkers to predict neonatal acute kidney injury. J Perinat Neonatal Nurs 2018;32(3):266-274. DOI: 10.1097/JPN.0000000000000295.

11. Shores DR, Everett AD. Children as biomarker orphans: progress in the field of pediatric biomarkers. J Pediatr 2017;193:14-20.e31. DOI: 10.1016/j.jpeds.2017.08.077.

12. Deirdre U. Sweetman, neonatal acute kidney injury - severity and recovery prediction and the role of serum and urinary biomarkers. Early Hum Dev 2017;105:57-61. DOI: 10.1016/ j.earlhumdev.2016.12.006.

13. Villa P, Jiménez $M$, Soriano $M C$, et al. Serum cystatin $C$ concentration as a marker of acute kidney dysfunction in critically ill patients. Crit Care 2005(2):139-143. DOI: 10.1186/cc3044.

14. Mussap M, Plebani M. Biochemistry and clinical role of human cystatin C. Crit Rev Clin Lab Sci 2004;41(5-6):467-550. DOI: 10.1080/10408360490504934.

15. Hameed HM, Sharbini SAE, Barakat NA, et al. Serum cystatin C is a poor biomarker for diagnosing acute kidney injury in critically-ill children. Indian J Criti Care Med 2013;17(2):92-98. DOI: 10.4103/09725229.114829 .

16. Shlipak MG, Matsushita K, Ärnlöv J, et al. Relationship of kidney function estimates to risks improvements by measuring serum cystatin C in blood. N Eng J Med 2013;369(10):932-943. DOI: 10.1056/ NEJMoa1214234.

17. Coll E, Botey A, Alvarez L, et al. Serum cystatin C as a new marker for non-invasive estimation of glomerular filtration rate and as a marker for early renal impairment. Am J Kidney Dis 2000;36(1):29-34. DOI: 10.1053/ajkd.2000.8237. 